\title{
A comprehensive view of focused practices
}

$\mathrm{N}$

early one-third of general practitioners in Canada run practices that focus on areas of special interest, a situation that would appear to be antithetical to the very concept of comprehensive care. Yet many claim it is just the opposite, suggesting family physicians with special skills provide patients with access to a wider range of services, though warning that too much specialization would indeed be a cause for concern.

"Within my own group of 11 doctors, seven have what might be called focused practices," says Dr. Cathy Scrimshaw, a family doctor in rural Alberta and an executive member of the Alberta College of Family Physicians. "They are still doing comprehensive family medicine and use those extra skills when we need them."

One of the general practitioners in Scrimshaw's practice in Pincher Creek, which serves a catchment area of about 10000 people, sometimes performs cesarian sections. Another does some anesthesia work. "The down side is that they are not in clinic as much," says Scrimshaw. "The upside is that we can provide a greater range of care."

Though conceding it is a "legitimate concern" that access to generalists could suffer if too many family doctors restrict the scope of their practices, Scrimshaw sees the trend of "specialist" general practitioners as being largely positive thus far. It reduces the burden on consultants and reduces wait times for some medical procedures. It can also improve job satisfaction for physicians.

"It offers something interesting and out of the ordinary from regular clinical work, maybe for one day a week," says Scrimshaw.

Of course, not all physicians believe the trend of increased specialization bodes well for the future of family medicine. In a study of perceptions of family medicine led by Dr. Marie-Dominique Beaulieu, a family physician and professor in the Department of Family Medicine at the Université of Montréal in

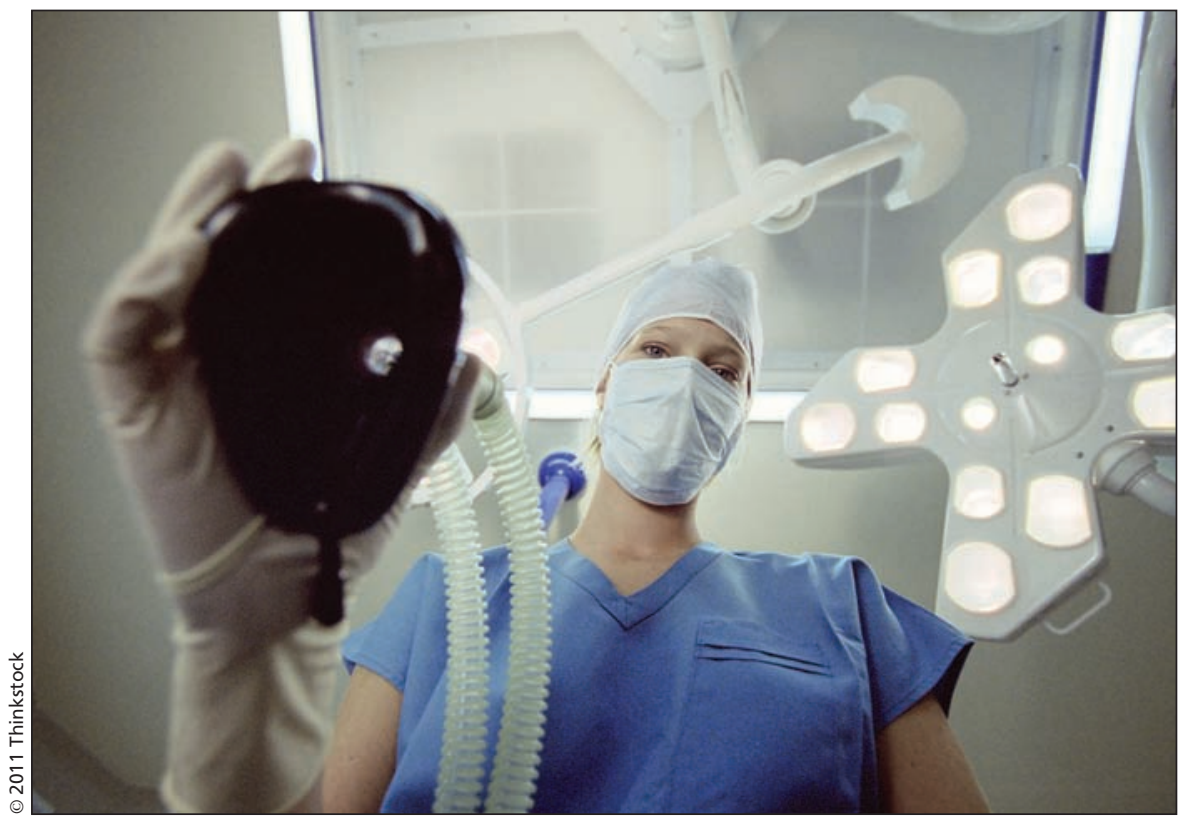

Some physicians believe that rather than limiting access to comprehensive care, general practitioners who pick up new skills in specialized areas, such as anesthesiology, increase the range of services they can provide to patients.

Quebec, some within the field suggested that the true generalist is becoming an endangered species (Soc Sci Med 2008; 67:1153-63). This trend not only restricts access to primary care, some participants said, but threatens the very profession of family medicine.

"Indeed, many family educator respondents believe specialization among family physicians represents a real danger to the profession's survival, because the health care system has a vital need for an integration function which, up to now, they have fulfilled," the paper states. "If they disappear, someone else will have to take their place."

The authors suggest, however, that models of comprehensive care must adapt to "ensure optimal use of available expertise" and, as such, the boundaries "between professional jurisdictions are thus subject to continued renegotiation." The paper concludes that promoting collaborative practice models may be the best strategy for "preserving the fundamental uniqueness of the profession of general practitioner."

It just so happens that team-based practices are where family doctors with specialized skills may benefit patients most, as doctors are able to refer patients to one another, says Dr. Cal Gutkin, executive director and chief executive officer of the College of Family Physicians of Canada.

Patients' access to some procedures would suffer if generalists didn't expand their skill sets because medical specialists are increasingly becoming subspecialists and even super-subspecialists, Gutkin adds. "There are gaps left in the more general areas of their specialties. ... Family physicians were the natural choice to move in and provide those services."

It is the hope of the college's leadership, however, that family doctors incorporate their newly acquired skills into their practices rather than focus solely on specialized services. One of the college's goals is to support physicians with special interests while encouraging them to stay within the umbrella of comprehensive care.

"From our perspective, identifying groups of physicians who have similar 
focuses or special interests is a positive thing. It will allow physicians to network, to get continuing medical education and to find a home with the college," says Dr. Rob Boulay, president of the College of Family Physicians of Canada. "From the perspective of communities, these physicians fulfill huge needs within the patient population that go unmet."

Do family physicians have ulterior motives, beyond meeting community needs, for specializing? Perhaps a financial incentive? They do indeed at least in the United States, according to Portland, Maine, family physician Dr. James Glazer, who has suggested that the financial and lifestyle benefits of specializing were a "compelling incentive" for medical graduates to "abandon" generalist medicine (Fam Pract Manag 2007;14:13-5).

"Many family physicians embark on careers in sports medicine, academic medicine or geriatrics in which they take no overnight call and have no responsibility for caring for hospitalized patients," he wrote. "As 'subspecialists,' they typically command higher rates of reimbursement for clinical work - particularly those with certificates of added qualification."

The same holds true in the United Kingdom, where a general practitioner with a special interest - or a GPwSI, as such a doctor is known there - commands a higher salary. This may explain, in part, why the number of family doctors choosing this path exceeds, by several thou- sand, the 1000 positions the National Health Service hoped to create by 2004 to free up consultants to concentrate on complicated patients. "For some GPs, the financial incentive can be one of the reasons, but remember this is not a gold mine," UK general practitioner Dr. Anita Sharma wrote in COPD in Primary Care (www.radcliffe-oxford.com /books/samplechapter/3168/Sharma _final_20-2023bbcrdz.pdf).

The increase in specialized family doctors in the UK has improved access to certain medical services, but it has come at a cost. The National Health Service pays almost twice as much when a patient visits a family doctor specializing in dermatology, for instance, instead of receiving outpatient care at the hospital, according to one study (BMJ 2005; doi:10.1136/bmj.38676.446910.7C.) "The main reason for these higher costs is that the patients attending the general practitioner with special interest service always see the relatively costly general practitioner with special interests," it states, "whereas outpatients might see the relatively costly consultant but may also see one of the consultant team (registrar or clinical assistant) who is less costly."

In Canada, however, there doesn't appear to be much to gain financially from dedicating a portion of a family practice to a special interest. Sure, family doctors receive higher fees for performing particular specialized procedures, such as cesarean sections, than for seeing people with the flu. But the increased patient volume seen by a generalist may offset the higher fees received by doctors focusing on particular services. In fact, a special interest such as geriatrics might even reduce a physician's income, because elderly patients often take more time to treat.

"I don't think that there is an actual financial benefit, probably more of a patient benefit," says Boulay.

Another reason special interests aren't likely to result in huge income increases is that most of them don't involve complex procedures, but rather fall within the traditional domain of primary care, says Dr. Tamara Buchel, president of the Manitoba College of Family Physicians. "Most are still well within the realm of comprehensive care," Buchel says. "Care of the elderly. Treating addictions. Those kinds of slightly enhanced competencies are still aspects of comprehensive care." — Roger Collier, CMAJ

CMAJ 2011. DOI:10.1503/cmaj.109-4040

Editor's note: Second of a three-part series

Part I: The changing face of family medicine

(www.cmaj.ca/lookup/doi/10.1503 /cmaj.109-4036).

Part III: Potential pitfalls of "specialized" primary care

(www.cmaj.ca/lookup/doi/10.1503 /cmaj.109-4044). 